Model predictive control of an AFE rectifier with dynamic references

by Daniel E. Quevedo, Ricardo P. Aguilera, Marcelo A. Pérez, Patricio Cortés, \& Ricardo Lizana

Copyright @ 2012 IEEE.

This is an author-prepared version of the article, reprinted from IEEE Transactions on Power Electronics, Vol. 27, Issue 7, p. 3128-3136.

http://ieeexplore.ieee.org/xpls/abs all.jsp?arnumber=6104162

This material is posted here with permission of the IEEE. Such permission of the IEEE does not in any way imply IEEE endorsement of any of University of Newcastle's products or services. Internal or personal use of this material is permitted. However, permission to reprint/republish this material for advertising or promotional purposes or for creating new collective works for resale or redistribution must be obtained from the IEEE by writing to pubs-permissions@ieee.org. By choosing to view this document, you agree to all provisions of the copyright laws protecting it. 


\title{
Model Predictive Control of an AFE Rectifier with Dynamic References
}

\author{
Daniel E. Quevedo, Member, IEEE, Ricardo P. Aguilera, Student Member, IEEE, Marcelo A. \\ Pérez, Member, IEEE, Patricio Cortés, Member, IEEE, \\ and Ricardo Lizana, Student Member, IEEE
}

\begin{abstract}
We present a finite control set model predictive controller for closed loop control of an active front-end rectifier. Our method operates in discrete-time and does not require additional modulators. The key novelty of the control algorithm presented lies in the way dynamic references are handled. The control strategy is capable of providing suitable references for the source active power and the rectified voltage, without requiring additional control loops. Experimental results show that fast and accurate tracking of dynamic $\mathrm{DC}$-voltage and reactive power references can be achieved, while respecting restrictions on maximum power levels of the rectifier.
\end{abstract}

\section{INTRODUCTION}

In many industrial applications active front-end rectifiers (AFEs) have emerged as an attractive topology. When compared to traditional diode-based rectifiers, AFEs allow one to obtain sinusoidal input currents with low harmonic distortion, whilst at the same time providing an regulated rectifier voltage. Moreover, the amount of reactive power drawn from the source can be manipulated in order to reach a unity power factor at the input (see, e.g., [2]-[4]), or also to compensate lack of reactive power in the source grid; see [5] and the references therein. Another area where AFEs play an important role is when a reduction of harmonic distortion is sought. In fact, this converter is of widespread use as an active filter in which case the AFE is connected in parallel to the non-linear load thereby generating the harmonic currents necessary to reduce the pollution in the source; see, e.g., [6].

For the control of AFEs, different methods based on pulsewidth modulation (PWM) techniques exist. The most popular algorithms use voltage-oriented control (VOC), and various forms of direct power control (DPC), see, e.g., [7]-[10]. The VOC approach works in the $\mathrm{d}-\mathrm{q}$ reference frame using two control loops: The external loop is based on a Proportional Integral (PI) controller which seeks to compensate the DCvoltage error by generating the direct current reference. The

This work has been presented in part at the IEEE Conference on Industrial Technology (ICIT 2010), see [1].

Daniel Quevedo and Ricardo Aguilera are with the School of Electrical Engineering \& Computer Science, The University of Newcastle, NSW 2308, Australia; e-mails: \{dquevedo, raguilera\}@ieee.org

Marcelo Pérez, Patricio Cortés and Ricardo Lizana, are with the Departamento de Electrónica, Universidad Técnica Federico Santa María, Valparaíso, Chile; emails: \{marcelo.perez, patricio.cortes, ricardo.lizana\}@usm.cl.

The research of Daniel Quevedo and of Ricardo Aguilera is supported under Australian Research Council's Discovery Projects funding scheme (project number DP110103074). Marcelo Pérez, Patricio Cortés and Ricardo Lizana acknowledge the support of the Chilean Research Council under grant Fondecyt 11090253, basal project FB0821 CCTVal and of the Universidad Técnica Federico Santa María. internal loop reduces the dq-current error by using two PI controllers. These generate dq-voltages which are utilized to produce the associated space vector modulation. DPC techniques also require two control loops, but in a different manner: With DPC, the external controller seeks to compensate the DCvoltage error by directly generating the power reference for an internal control loop. In the standard approach, as described, e.g., in [7], the switching actions are obtained from a table lookup, which takes into account not only the DC-voltage error, but also active and reactive power estimates. Other types of DPC are based on the use of external modulators, see [8][10].

The main drawback of both VOC and DPC methods is the need for local linearizations for the linear control design part. The latter issue was investigated recently in [11].

Over the last decade, model predictive control (MPC) strategies have emerged as a promising control technique for power electronics applications; see, e.g., [12]-[16]. The main advantage of these predictive strategies, when compared to traditional PWM methods, derive from the fact that switching effects can explicitly be considered without approximations. For example, so called Finite Control Set MPC formulations (sometimes also referred to as Direct MPC, see [17]) have been presented, for example, in [18]-[20]. Here a switching model of the converter is used to minimize a running cost function through exploration of the different switch combinations. The switching action to be applied at the next sampling instant is that which minimizes the cost function. The latter can be chosen to reflect various control objectives. In addition to input currents, one can focus on other issues including current spectra [21] and number of commutations; see, e.g., [22].

An MPC-based direct power control method for AFEs was presented in [23]. A key issue which arises when controlling an AFE is that, unavoidably, DC-voltage and active power levels are coupled. Thus, is is necessary to find compatible references for these variables. For that purpose, in [23] the active power reference is obtained from an external PI-controller which is designed to compensate DC-voltage errors. The predictive controller then provides switching actions to track the desired active and reactive power references at the input. Not surprisingly, tuning the external PI controller needed in [23] becomes a difficult task, due to the discrete-time switching nature of the converter.

The present work proposes an MPC formulation for closed loop control of AFEs. The key novelty of our method is that it is capable of providing suitable references for the source active 


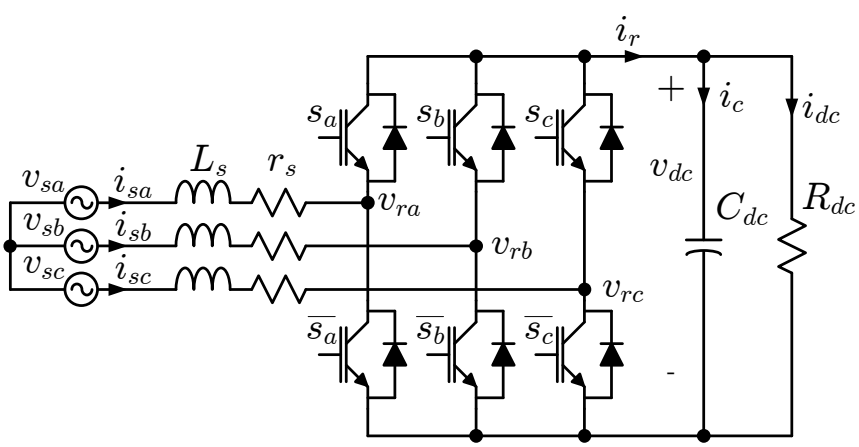

Figure 1. Active front-end rectifier with floating neutral point

power and the rectified voltage, without requiring additional control loops. The method presented is formulated in discretetime, uses a state-space model of the converter and directly provides the switching action to be applied. As documented by experimental results on a lab-prototype of 500[W], our formulation allows one to incorporate restrictions on maximum power levels, without incurring any loss of performance due to windup issues, which are typical in PI-control loops, see also [24].

The remainder of this manuscript is organized as follows: In Section II we give a dynamic model of the AFE rectifier. Section III presents the cost function chosen. In Section IV we show how a compatible reference can be formulated. Simulation studies and experimental results are included in Section V. Section VI draws conclusions.

\section{AFE RECTIFIER}

In the present work, we focus on the AFE rectifier shown in Fig. 1. As can be seen in that figure, the rectifier is a threephase fully-controlled bridge consisting of 6 power transistors connected to a three-phase power source $v_{s}$ by means of a filter. The latter is represented by an inductance $L_{s}$ and the parasitic resistance $r_{s}$. The neutral point is electrically floating.

\section{A. Continuous Time Model}

We will adopt an $a b c$-frame, as presented in [25], and suppose that the three-phase source voltages $v_{s a}, v_{s b}$ and $v_{s c}$ are symmetric, so that in the AFE, we have:

$$
\begin{aligned}
v_{s a}(t)+v_{s b}(t)+v_{s c}(t) & =0 \\
i_{s a}(t)+i_{s b}(t)+i_{s c}(t) & =0
\end{aligned}
$$

at all times $t \in \mathbb{R}$. Thus, the source current obeys

$$
\begin{aligned}
\frac{d i_{s a}(t)}{d t}= & \frac{1}{L_{s}} v_{s a}(t)-\frac{r_{s}}{L_{s}} i_{s a}(t) \\
& \quad-\frac{1}{3 L_{s}}\left(2 s_{a}(t)-s_{b}(t)-s_{c}(t)\right) v_{d c}(t) \\
\frac{d i_{s b}(t)}{d t}= & \frac{1}{L_{s}} v_{s b}(t)-\frac{r_{s}}{L_{s}} i_{s b}(t) \\
& \quad-\frac{1}{3 L_{s}}\left(-s_{a}(t)+2 s_{b}(t)-s_{c}(t)\right) v_{d c}(t),
\end{aligned}
$$

for all $t \in \mathbb{R}$ and where the switch variables $s_{a}(t), s_{b}(t)$ and $s_{b}(t)$ are equal to 1 , if at time $t$ the associated switch is conducting, and equal to zero, if it is blocking current. Consequently, the active source power can be expressed via:

$$
P_{s}(t)=2\left(v_{s a}(t) i_{s a}(t)+v_{s b}(t) i_{s b}(t)\right) .
$$

To obtain a dynamic model for the rectified voltage $v_{d c}(t)$, we note that the currents on the rectifier side are described via:

$$
\begin{aligned}
i_{r}(t) & =\left(s_{a}(t)-s_{c}(t)\right) i_{s a}(t)+\left(s_{b}(t)-s_{c}(t)\right) i_{s b}(t) \\
i_{d c}(t) & =\frac{v_{d c}(t)}{R_{d c}} \\
i_{c}(t) & =i_{r}(t)-i_{d c}(t) .
\end{aligned}
$$

Consequently, the dynamics of $v_{d c}(t)$ is characterized via:

$$
\begin{gathered}
\frac{d v_{d c}(t)}{d t}=\frac{1}{C_{d c}}\left(\left(s_{a}(t)-s_{c}(t)\right) i_{s a}(t)+\left(s_{b}(t)-s_{c}(t)\right) i_{s b}(t)\right. \\
\left.-\frac{1}{R_{d c}} v_{d c}(t)\right), \quad \forall t \in \mathbb{R} .
\end{gathered}
$$

Finally, the active power in the rectifier side can be expressed as follows:

$P_{r}(t)=\left(\left(s_{a}(t)-s_{c}(t)\right) i_{s a}(t)+\left(s_{b}(t)-s_{c}(t)\right) i_{s b}(t)\right) v_{d c}(t)$.

\section{B. Discrete Time Model}

The MPC algorithm to be developed operates in discrete time with fixed sampling period $h>0$. To obtain a discrete time model of the system, we introduce

$$
\mathbf{i}_{s}(k) \triangleq\left[\begin{array}{l}
i_{s a}(k) \\
i_{s b}(k)
\end{array}\right], \quad \mathbf{v}_{s}(k) \triangleq\left[\begin{array}{c}
v_{s a}(k) \\
v_{s b}(k)
\end{array}\right], \quad \mathbf{s}(k) \triangleq\left[\begin{array}{c}
s_{a}(k) \\
s_{b}(k) \\
s_{c}(k)
\end{array}\right],
$$

where $k \in \mathbb{N}$ refers to the sampling instants $k h$. An Euler approximation of the continuous time model represented by (3) and (6) then provides: ${ }^{1}$

$$
\begin{aligned}
\mathbf{i}_{s}(k+1) & =\left(1-\frac{r_{s} h}{L_{s}}\right) \mathbf{i}_{s}(k)+\frac{h}{L_{s}}\left(\mathbf{v}_{s}(k)-\mathbf{M s}(k) v_{d c}(k)\right) \\
v_{d c}(k+1) & =\left(1-\frac{h}{C_{d c} R_{d c}}\right) v_{d c}(k)+\frac{h}{C_{d c}}(\mathbf{s}(k))^{T} \mathbf{F} \mathbf{i}_{s}(k),
\end{aligned}
$$

where $k \in \mathbb{N}$ and where

$$
\mathbf{M} \triangleq \frac{1}{3}\left[\begin{array}{ccc}
2 & -1 & -1 \\
-1 & 2 & -1
\end{array}\right], \quad \mathbf{F} \triangleq\left[\begin{array}{cc}
1 & 0 \\
0 & 1 \\
-1 & -1
\end{array}\right]
$$

If we now introduce the state-vector of the AFE rectifier,

$$
x(k) \triangleq\left[\begin{array}{c}
\mathbf{i}_{s}(k) \\
v_{d c}(k)
\end{array}\right]
$$

\footnotetext{
${ }^{1}$ Since the switch combinations are held constant between updates, the AFE is linear time-varying where the system matrices are held constant between updates. Due to the simplicity of the model, and since the time constants are much larger than the sampling interval chosen, se Section V, using an Euler approximation gives good results.
} 
then the model (8) can be written in compact form via:

$$
x(k+1)=A_{\mathbf{s}(k)} x(k)+B \mathbf{v}_{s}(k), \quad k \in \mathbb{N}
$$

where: ${ }^{2}$

$$
A_{\mathbf{s}(k)} \triangleq\left[\begin{array}{cc}
\left(1-\frac{r_{s} h}{L_{s}}\right) I_{2} & -\frac{h}{L_{s}} \mathbf{M s}(k) \\
\frac{h}{C_{d c}}(\mathbf{s}(k))^{T} \mathbf{F} & 1-\frac{h}{C_{d c} R_{d c}}
\end{array}\right], \quad B \triangleq\left[\begin{array}{c}
\frac{h}{L_{s}} I_{2} \\
0_{1 \times 2}
\end{array}\right] .
$$

Remark 1 (Finite-set constraints): It is worth noting that, each switch variable at time $k$, namely $\mathbf{s}(k)$, only takes values in the finite set

$$
\mathcal{S} \triangleq\left\{\left[\begin{array}{l}
0 \\
0 \\
0
\end{array}\right],\left[\begin{array}{l}
0 \\
0 \\
1
\end{array}\right],\left[\begin{array}{l}
0 \\
1 \\
0
\end{array}\right],\left[\begin{array}{l}
0 \\
1 \\
1
\end{array}\right],\left[\begin{array}{l}
1 \\
0 \\
0
\end{array}\right],\left[\begin{array}{l}
1 \\
0 \\
1
\end{array}\right],\left[\begin{array}{l}
1 \\
1 \\
0
\end{array}\right],\left[\begin{array}{l}
1 \\
1 \\
1
\end{array}\right]\right\}
$$

Expression (12) then shows that the system matrix $A_{\mathbf{s}(k)}$ is also finite-set constrained, i.e., we have that $A_{\mathbf{s}(k)} \in \mathcal{A}$ for a given set $\mathcal{A}$ having eight elements. Discrete-time control design then amounts to choosing the sequence of switch values $\mathbf{s}(k) \in \mathcal{S}, k \in \mathbb{N}$ or, equivalently, selecting the sequence of system matrices $A_{\mathbf{s}(k)} \in \mathcal{A}$ for all $k \in \mathbb{N}$.

In the following section, we will present a model predictive control strategy for the AFE rectifier, based on the model given in (11).

\section{Model Predictive Control of the AFE}

Model predictive control is based upon online optimization of a performance index for current system state and future reference trajectories; see, e.g., [26]-[28]. To obtain a practical method which does not require excessive computation times, in the present work we adopt a cost function which only evaluates the effect of the switch decisions to be made at current time instant. This is computationally attractive and, as will become apparent in Section V, gives good performance. The cost function presented next quantifies a quadratic norm of the tracking error of the three components of the state vector at the next time-instant. ${ }^{3}$

From an electrical viewpoint, the main purpose of the AFE is to provide a DC-voltage to the load, whilst at the same time managing a desired balance between active and reactive power. According to the state-space model introduced in Section II-B, for a given system state $x(k)$, the system state, which would result if at time $k$, the switches $s_{a}, s_{b}$ and $s_{c}$ were set to $\mathbf{s}(k) \in \mathcal{S}$ is given by:

$$
x^{\prime}(k+1)=A_{\mathbf{s}(k)} x(k)+B \mathbf{v}_{s}(k) .
$$

By approximating $\mathbf{v}_{s}(k+1)=\mathbf{v}_{s}(k)$, we obtain that the

\footnotetext{
${ }^{2} I_{2}$ denotes the $2 \times 2$ identity matrix, whereas $0_{1 \times 2}=\left[\begin{array}{ll}0 & 0\end{array}\right]$ and the superscript ${ }^{T}$ refers to transposition.

${ }^{3}$ Most applications of MPC in power electronics and drives use such horizon-one cost functions [12]. Interestingly, in some situations, the use of horizon one also gives the optimal solution to a formulation with a larger horizon, see [29], [30].
}

associated predicted active power satisfies:

$$
\begin{aligned}
P_{s}^{\prime}(k+1) & =\mathbf{v}_{s}^{T}(k)\left[\begin{array}{ll}
2 & 1 \\
1 & 2
\end{array}\right] \mathbf{i}_{s}^{\prime}(k+1) \\
& =\mathbf{v}_{s}^{T}(k)\left[\begin{array}{lll}
2 & 1 & 0 \\
1 & 2 & 0
\end{array}\right] x^{\prime}(k+1),
\end{aligned}
$$

whereas

$$
\begin{aligned}
Q_{s}^{\prime}(k+1) & =\mathbf{v}_{s}^{T}(k) \sqrt{3}\left[\begin{array}{cc}
0 & 1 \\
-1 & 0
\end{array}\right] \mathbf{i}_{s}^{\prime}(k+1) \\
& =\mathbf{v}_{s}^{T}(k)\left[\begin{array}{ccc}
0 & \sqrt{3} & 0 \\
-\sqrt{3} & 0 & 0
\end{array}\right] x^{\prime}(k+1)
\end{aligned}
$$

is the predicted reactive power, and

$$
v_{d c}^{\prime}(k+1)=\left[\begin{array}{lll}
0 & 0 & 1
\end{array}\right] x^{\prime}(k+1),
$$

the predicted DC-voltage.

The main control objective in an AFE rectifier is to transfer active power from the source, $P_{s}$, to the DC-load. To do this, in modulation-based strategies where the modulation index is the control input, it is common to equalize the average active power source $P_{s}(t)$ with the average rectifier power, $P_{r}(t)$. This procedure then leads to a relationship between $P_{s}(t)$ and the DC-voltage, $v_{d c}(t)$, see [25]. However, it is in general not possible to set $P_{s}(t)=P_{r}(t)$ at all instants. For example, when the power switches take the same value, say, $S_{a}(t)=S_{b}(t)=S_{c}(t)$, then the input and output side are decoupled. In this case, as shown in (7), the rectifier power is clearly null, $P_{r}(t)=0$, while the source power presented in (4) may present a different value $P_{s}(t) \neq 0$. This motivates us to introduce a so-called filtered DC-voltage reference $\tilde{v}_{d c}^{\star}(t)$, which allows us to establish a relationship between an average value of these two variables.

The controller proposed in the present work uses the above prediction model for current state $x(k)$ to choose the switching values $\mathbf{s}(k)$ which minimize a cost function of the form: ${ }^{4}$

$$
\begin{aligned}
J(\mathbf{s}(k))=\frac{1}{\bar{v}_{d c}^{2}}\left(\tilde{v}_{d c}^{\star}(k+1)-v_{d c}^{\prime}(k+1)\right)^{2} \\
+\frac{k_{p}}{\bar{P}^{2}}\left(P_{s}^{\star}(k+1)-P_{s}^{\prime}(k+1)\right)^{2} \\
+\frac{k_{q}}{\bar{P}^{2}}\left(Q_{s}^{\star}(k+1)-Q_{s}^{\prime}(k+1)\right)^{2} .
\end{aligned}
$$

In (18), the superscript ${ }^{\star}$ refers to reference values; $k_{p}$ and $k_{q}$ are tuning parameters, which allow the designer to trade capacitor voltage reference deviations for deviations in active and reactive power. It is important to have in mind that in the cost function, the three system states are normalized by the factors $\overline{v_{d c}}$ and $\bar{P}$, thus, providing comparable deviation errors. For our results, see Section V, we chose $k_{p}=k_{q}=$ 1 in which case the controller gives equal weight to all the deviation components.

The cost function in (18) uses $Q_{s}^{\star}(k+1), P_{s}^{\star}(k+1)$, and also a filtered DC-voltage reference $\tilde{v}_{d c}^{\star}(k+1)$. In our formulation,

\footnotetext{
${ }^{4}$ In our formulation, we assume that computation times are negligible when compared to the sampling period. This reflects the fact that, in our experimental platform, computations take $3[\mu s]$, whereas the sampling period is taken as $h=20[\mu s]$.
} 
we assume that the reference value $Q_{s}^{\star}(k+1)$ and also a reference for the DC-voltage, say $v_{d c}^{\star}(k+1)$, are given. In the following section, we will show how to obtain $P_{s}^{\star}(k+1)$ and $\tilde{v}_{d c}^{\star}(k+1)$ from $v_{d c}^{\star}(k+1)$ and $Q_{s}^{\star}(k+1)$. The aim is to design references which are consistent from an electrical viewpoint and when used in (18) allow the controller to give good performance, despite system constraints.

It is worth recalling that in standard control formulations for AFEs, it is common to only control the average reactive power, $Q_{s}(t)$, and the DC-voltage, $v_{d c}(t)$ due to the fact that, as seen above, the latter is coupled to the average active source power $P_{s}(t)$. In contrast, in the present formulation, we are interested in tracking a dynamic filtered reference from the instantaneous values of the system state variables. For that purpose we include the active power source, $P_{s}(k)$, in the cost function (18). This also allows us to incorporate a safety constraint for the power source, $P_{s}(k) \leq P^{\max }$, in order to avoid over currents in the AC side.

Remark 2 (Plant State Weighting): The choice made in (18) amounts to weighting a quadratic form of the tracking error of the predicted state vector. Many theoretical results on MPC algorithms for systems without finite-set constraints suggest that such a formulation will often lead to closed loops having favorable stability and performance features; see, e.g., [26]-[28], [31], [32]. The case of systems with finite input constraints was studied in [33], [34]. How to extend these results to the present situation where the system matrix is finite-set constrained, see Remark 1, remains an open, and certainly non-trivial, problem.

\section{REFERENCE DESIGN}

As noted in the introduction, a key difficulty when controlling an AFE lies in that successful tracking of power and voltage references cannot be achieved for arbitrarily chosen and time-varying reference signals. In fact, active power $P_{s}(k)$ and DC-voltage $v_{d c}(k)$ are unavoidably coupled. This relationship is difficult to characterize exactly in closed form, since it depends not only upon electrical parameters of the system, but also upon the switching law used.

One way to find compatible reference values has been explored recently in [23], where the use of an additional PI-control loop was examined. It turns out that PI-control gives perfect tracking of constant references in steady state. However, tuning the external controller becomes a difficult task, since the AFE is a discrete-time switching system.

We will next present an alternative method to provide suitable references for the source power and the rectified voltage. Our formulation uses directly predictive control concepts and does not require an additional control loop. ${ }^{5}$ Furthermore, the method proposed allows one to incorporate restrictions on maximum power levels, without any integrator windup problems.

\section{A. Design of Compatible References}

Given references $v_{d c}^{\star}(k)$ and $Q_{s}^{\star}(k)$ and the current system state $x(k)$, the reference design problem considered consists

${ }^{5}$ To some extent, our approach is related to ideas underlying so-called reference governors for model predictive control formulations; see, e.g., [35].

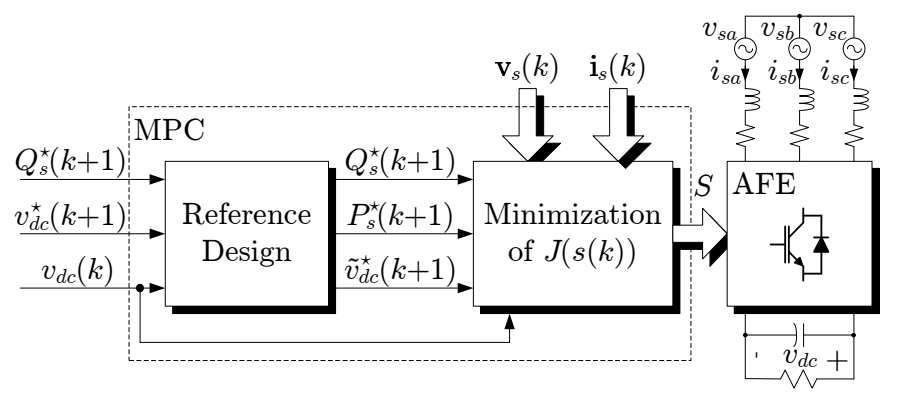

Figure 2. MPC with Dynamic Reference Design

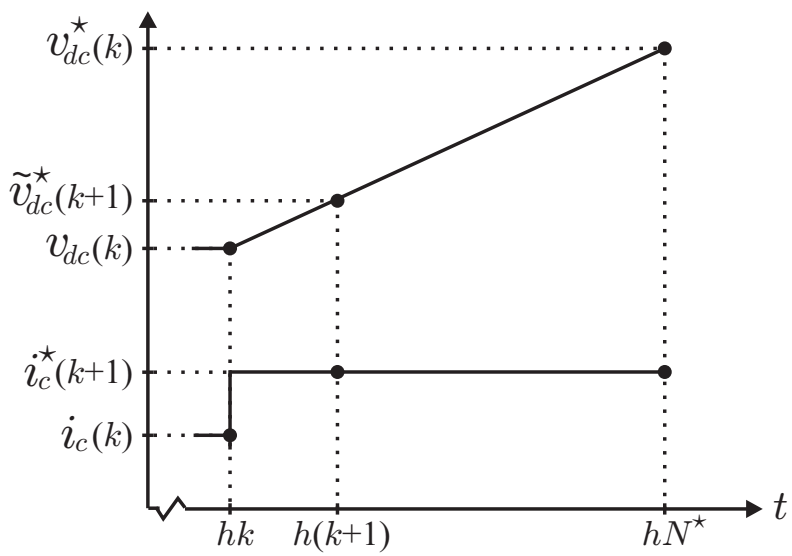

Figure 3. Dynamic reference design: Capacitor current $i_{c}^{\star}$ needed to increase the capacitor voltage $v_{d c}$ in order to reach the reference $v_{d c}^{\star}$ in $N^{\star}$ time steps.

in finding a compatible reference for $P_{s}(k+1)$ and an associated filtered reference value $\tilde{v}_{d c}^{\star}(k+1)$ as used in the cost function (18), see also Fig. 2. For that purpose, it is convenient to examine some electrical properties inherent to the AFE topology.

We first note that the capacitor voltage $v_{d c}$ can only be adjusted by the capacitor current $i_{c}$. Since the latter quantity cannot be made arbitrarily large, we will introduce a reference prediction horizon, say $N^{\star}$. This horizon value constitutes a design parameter which determines the filtered reference $\tilde{v}_{d c}^{\star}(k+1)$. More specifically, $\tilde{v}_{d c}^{\star}(k+1)$ is obtained from $v_{d c}^{\star}(k)$ and $v_{d c}(k)$ via

$$
\tilde{v}_{d c}^{\star}(k+1)=v_{d c}(k)+\frac{1}{N^{\star}}\left(v_{d c}^{\star}(k)-v_{d c}(k)\right),
$$

which amounts to allowing the converter to reach $v_{d c}^{\star}(k)$ linearly in $N^{\star}$ steps, see Fig. 3.

Having calculated $\tilde{v}_{d c}^{\star}(k+1)$, we next seek to find a compatible reference for the active input power. Here, it is important to recall that the capacitor current $i_{c}$ needs to ultimately be provided by the AC-source and thereby affects the active input power $P_{s}$. To be more precise, the filtered reference $\tilde{v}_{d c}^{\star}(k+1)$ in (19) requires a capacitor current value 
of

$$
\begin{aligned}
i_{c}^{\star}(k+1) & =\frac{C_{d c}}{h}\left(\tilde{v}_{d c}^{\star}(k+1)-v_{d c}(k)\right), \\
& =\frac{1}{N^{\star}}\left(\frac{C_{d c}}{h}\left(v_{d c}^{\star}(k+1)-v_{d c}(k)\right)\right) .
\end{aligned}
$$

Thus, the capacitor current, $i_{c}$, is limited to a $\left(100 / N^{\star}\right) \%$ of the total current required to lead the DC-voltage, $v_{d c}$, to its desired reference $v_{d c}^{\star}$. This reduced capacitor current, in turn, necessitates a converter current, say $i_{r}^{\star}(k+1)$, given by:

$$
i_{r}^{\star}(k+1)=i_{c}^{\star}(k+1)+\frac{1}{2 R_{d c}}\left(v_{d c}(k)+\tilde{v}_{d c}^{\star}(k+1)\right) .
$$

Therefore, the overall rectifier power needed to track $\tilde{v}_{d c}^{\star}(k+1)$ satisfies:

$$
\begin{aligned}
P_{r}^{\star}(k+1)= & \tilde{v}_{d c}^{\star}(k+1) i_{r}^{\star}(k+1) \\
= & \left(\frac{1}{2 R_{d c}}+\frac{C_{d c}}{h}\right)\left(\tilde{v}_{d c}^{\star}(k+1)\right)^{2} \\
& +\left(\frac{1}{2 R_{d c}}-\frac{C_{d c}}{h}\right) \tilde{v}_{d c}^{\star}(k+1) v_{d c}(k),
\end{aligned}
$$

where we have used (20) and (22).

As noted before, the overall rectifier power needs to be provided by the AC-source. To obtain a value for the corresponding active input power reference, namely $P_{s}^{\star}(k+1)$, we will consider only the fundamental component of the alternating source currents. By taking into account the power losses which occur in the inductor resistances $r_{s}$, we obtain that compatible power references are (approximately) related by:

$$
P_{s}^{\star}(k+1)=\frac{2 r_{s}}{3 \hat{V}_{s}^{2}}\left(P_{s}^{\star}(k+1)\right)^{2}+P_{r}^{\star}(k+1),
$$

where $\hat{V}_{s}$ is the source voltage amplitude. The solution to (24) gives the desired reference for $P_{s}(k+1)$, namely:

$$
P_{s}^{\star}(k+1)=\frac{3 \hat{V}_{s}^{2}}{4 r_{s}}\left(1-\sqrt{1-\frac{8 r_{s}}{3 \hat{V}_{s}^{2}} P_{r}^{\star}(k+1)}\right),
$$

where $P_{r}^{\star}(k+1)$ is as in (23).

To summarize, the references used in the cost function (18) are obtained though expressions (19) and (25). It is worth emphasizing that the reference values $P_{s}^{\star}(k+1)$ and $\tilde{v}_{d c}^{\star}(k+1)$ are calculated at each time instant $k$ for a given plant state $x(k)$ and references $v_{d c}^{\star}(k)$ and $Q_{s}^{\star}(k)$. This allows the controller to track dynamic references. The reference prediction horizon $N^{\star}$ used in (19) serves to trade-off response times versus control effort by limiting the total increment in the capacitor current, $i_{c}$, as presented in (21). In fact, if a faster tracking response is desired, then $N^{\star}$ should be chosen small. However, this will, in general, lead to large converter currents. To incorporate current limitations, one can adopt the embellishments of the basic control algorithm presented in Section IV-B below.

Remark 3: The reference prediction horizon $N^{\star}$ slides forward in time in a moving horizon manner. Viewed from this perspective, the proposed control algorithm can be regarded as having a unit switching horizon, but an overall prediction horizon equal to $N^{\star}$, compare to ideas expressed in [22]. As will be apparent by the experimental results included in Section V, our formulation gives good performance, but requires only a moderate computational effort.

\section{B. Incorporation of current limits}

To avoid providing reference values which are associated with large converter currents, one can limit $P_{s}^{\star}(k+1)$ in (25) according to:

$$
\left|P_{s}^{\star}(k+1)\right| \leq P^{\max }(k+1),
$$

where

$$
P^{\max }(k+1) \triangleq \sqrt{\left(3 \hat{V}_{s} \hat{I}_{s}^{\max } / 2\right)^{2}-\left(Q_{s}^{\star}(k+1)\right)^{2}}
$$

and $\hat{I}_{s}^{\max }$ is a limit for the components of $\mathbf{i}_{s}(k)$.

In addition, one can also restrict the possible switch combinations $\mathbf{s}(k)$ when minimizing the cost function $J(\mathbf{s}(k))$ in (18) to those $\mathbf{s}(k) \in \mathcal{S}$, which provide current predictions $\mathbf{i}_{s}^{\prime}(k+1)$ satisfying the limit. The proposed modifications can be expected to lead to control loops which are not affected by windup problems typical of PI-control loops, see [36] for related discussions on the use of MPC for linear-time invariant systems with convex constraints.

\section{Results}

To verify the performance of the predictive control strategy proposed in the present work, simulation studies and experiments were carried out. Simulations were performed using Matlab/Simulink software. The lab prototype of the AFE rectifier, presented in Fig. 1, is comprised of a 6-pack IGBT module. The electrical parameters of the system are given by $r_{s}=0.4[\Omega], L_{s}=15[m H], C_{d c}=1500[u F]$ and $R_{d c}=60[\Omega]$. To protect the power device, the source current is limited to a maximum of $\hat{I}_{s}^{\max }=8[A]$. In addition, the source voltage is chosen to be $\hat{V}_{s}=62[\mathrm{~V}]$, whereas its frequency is $f_{o}=50[\mathrm{~Hz}]$. This voltage is obtained from the grid $(220[\mathrm{~V}] \mathrm{rms})$ and adapted by using an auto-transformer. It is important to emphasize that the grid voltage, $v_{s}$, contains $4.5 \%$ of fifth harmonic and a total harmonic distortion of $5 \%$. This amount of fifth harmonic is taken into account in the simulation tests, which thereby exhibit a similar system behaviour to that observed in the experiments.

The algorithm of Sections III and IV (including the modification presented in Section IV-B), was implemented in a digital platform using a Xilinx Spartan 3 FPGA considering a sampling period of $h=20[\mu s]$. The weighting factors in the cost function $J(\mathbf{s}(k))$ were chosen as $k_{p}=k_{q}=1$ and the reference prediction horizon was set as $N^{\star}=320$. This allows the controller to limit the capacitor current increment to about $3 \%$ of the total current needed to lead the DCvoltage to its desired reference. To capture the required data, an Agilent DSO5014A oscilloscope was used. It is important to emphasize that control calculations perfomed in the digital platform require less than $3[\mu s]$. Therefore, the optimal switch combination is applied to the converter with this delay, and before the following sampling instant. 


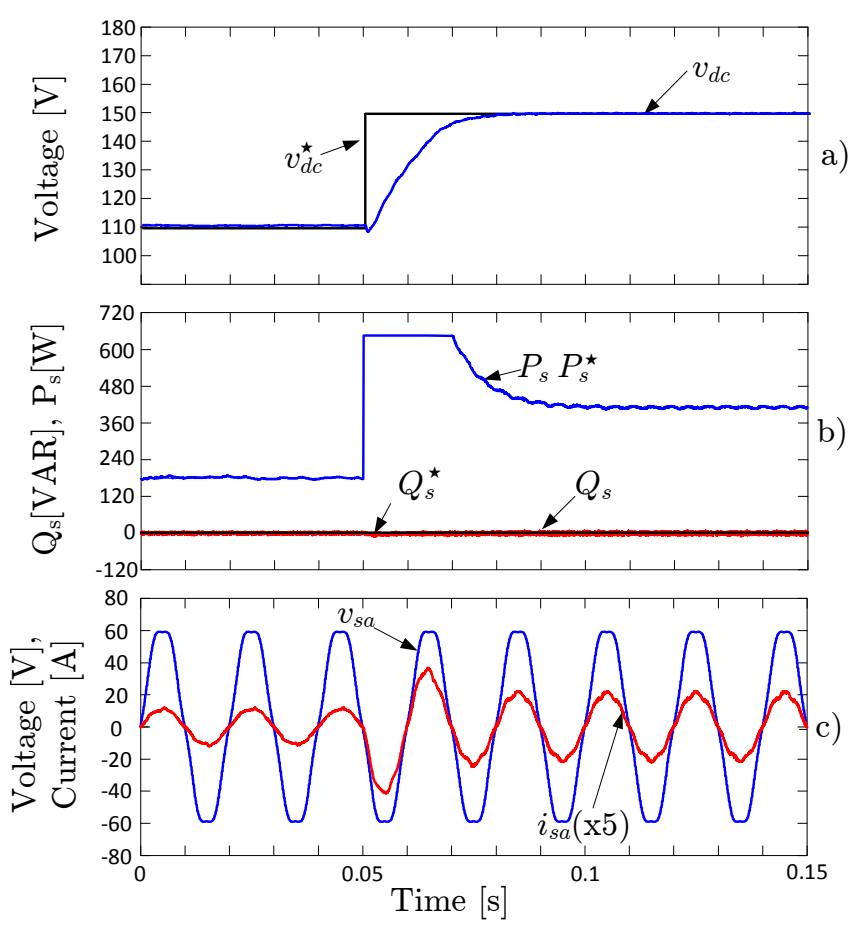

Figure 4. Performance of the MPC algorithm presented - Simulation: step in the (unfiltered) DC-voltage reference $v_{d c}^{\star}$.

\section{A. DC-Voltage Tracking}

We first investigate DC-voltage tracking. The performance of the proposed predictive strategy when a change in the DC-voltage reference $v_{d c}^{\star}$ is produced is depicted in Figs. 4 and 5. The initial DC-voltage is taken as $v_{d c}=110[\mathrm{~V}]$, corresponding to a source power of the order of $P_{s}=180[\mathrm{~W}]$. A unity power factor is desired, which results in null reactive power, i.e., $Q_{s}^{\star}=0[\mathrm{VAr}]$. As can be clearly appreciated in the figures, the predictive controller accommodates this requirement, by making the source current $i_{s a}$ be in phase with the source voltage $v_{s a}$.

To examine the effect of the tuning parameters on the AFE behaviour, we performed the same test but considering a smaller weighting factor for the reactive power, namely, $k_{q}=0.01$. This result is shown in Fig. 6. Here, it can be appreciated that the reactive power exhibits a higher ripple during steady state when compared to the previous case. Additionally, it can be noticed that during the transient the increment in the reactive power error is considerable higher than in the previous case. Henceforth, due to the good performance obtained, in the remaining tests the tuning factors will be kept at their original values, namely, $k_{p}=k_{q}=1$.

At time instant $t=0.05[s]$, the DC-voltage reference is increased to $v_{d c}^{\star}=150[V]$. This voltage increment requires active power from the source. However, the latter power is limited to $P_{\max }=650[\mathrm{~W}]$ due to the fact that, as mentioned above, the current that the AFE prototype can manage is limited to $I_{\max }=\hat{I}_{s}^{\max }=8[A]$. It can be noticed that both simulated and practical results illustrate a similar system behavior, despite the presence of the measurement noise. In particular, in both cases the system takes about $20[\mathrm{~ms}]$ to

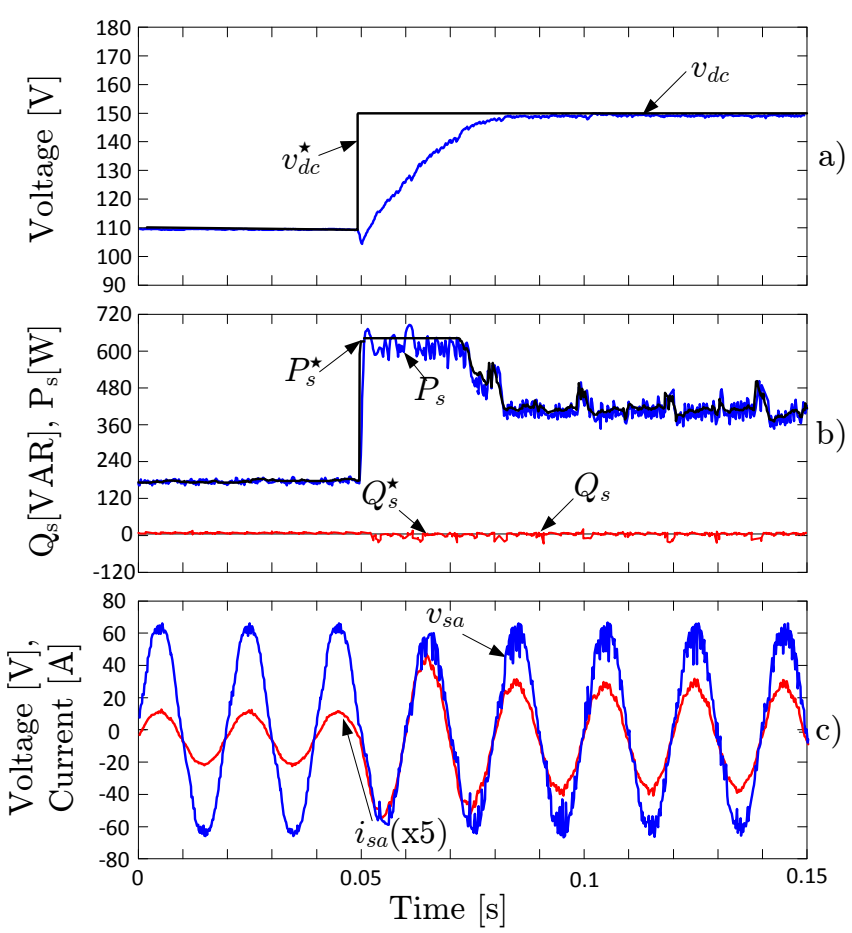

Figure 5. Performance of the MPC algorithm presented - Experiment: step in the (unfiltered) DC-voltage reference $v_{d c}^{\star}$.

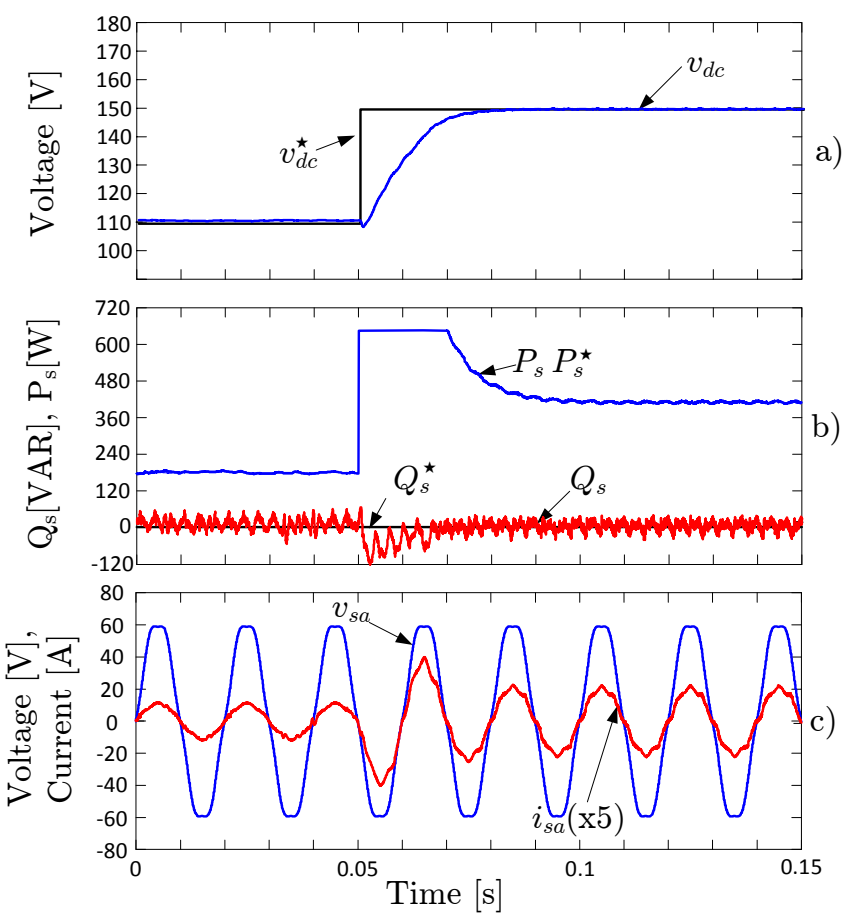

Figure 6. Performance of the MPC algorithm presented - Simulation: step in the (unfiltered) DC-voltage reference $v_{d c}^{\star}$. $\left(k_{q}=0.01\right)$.

reach the new DC-voltage reference. It can also be appreciated in Figs. 4 and 5 that the proposed controller gives good tracking performance, with no overshoot. It is remarkable that throughout the transient where saturations occur, the reactive power $Q_{s}$ has been held approximately equal to zero by the controller, keeping the source current in phase with its 


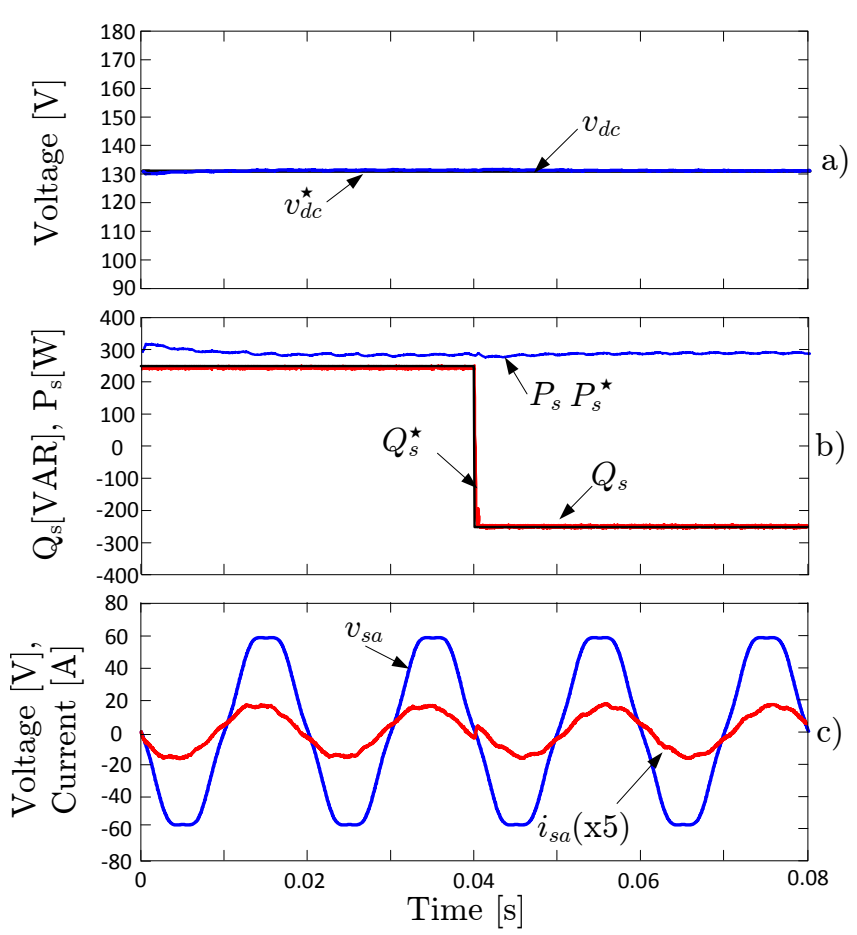

Figure 7. Performance of the MPC algorithm presented - Simulation: step in the reactive power reference $Q_{s}^{\star}$.

associated voltage. The total harmonic distortion in the source current for the initial condition, $v_{d c}=110[\mathrm{~V}]$, is $\mathrm{THD}_{i}=5 \%$. For a DC-voltage of $v_{d c}=150[V]$ the obtained distortion is $\mathrm{THD}_{i}=5 \%$. It is important to emphasize that the current distortion is also affected by the distortion in the voltage source which is given by $\mathrm{THD}_{v}=5 \%$.

\section{B. Tracking of Dynamic Reactive Power References}

One of the main uses of AFE converters is to compensate for the lack of reactive power of the electrical grid. Therefore, it is important to verify that the proposed controller can maintain the rectified DC-voltage even when different power factors are demanded of the system. Figs. 7 and 8 illustrate the achieved performance when facing this situation. Similar to the test documented in Section V-A, the initial condition of the system considers a DC-voltage of $v_{d c}=130[\mathrm{~V}]$ and an active power in the order of $P_{s}=300[W]$. However, reactive power $Q_{s}=250[\mathrm{VAr}]$ is provided from the rectifier to the source, amounting to a positive power factor of $\mathrm{PF}=0.769$. Consequently, and as can be seen in the figures, the source current lags the source voltage by about $39.7^{\circ}$.

To examine the tracking capabilities of the system, at approximately $t=0.04[s]$, a step down change in the reactive power reference is introduced, from $Q_{s}=+250[\mathrm{VAr}]$ to $Q_{s}=-250$ [VAr]. It can be appreciated in Figs. 7 and 8 that the controller makes the AFE track this step change quickly, barely affecting the active power $P_{s}$ or the capacitor voltage $v_{d c}$. In steady state, the power factor is negative ( $\mathrm{PF}=-0.769)$, the source current leading the source voltage by $39.7^{\circ}$.

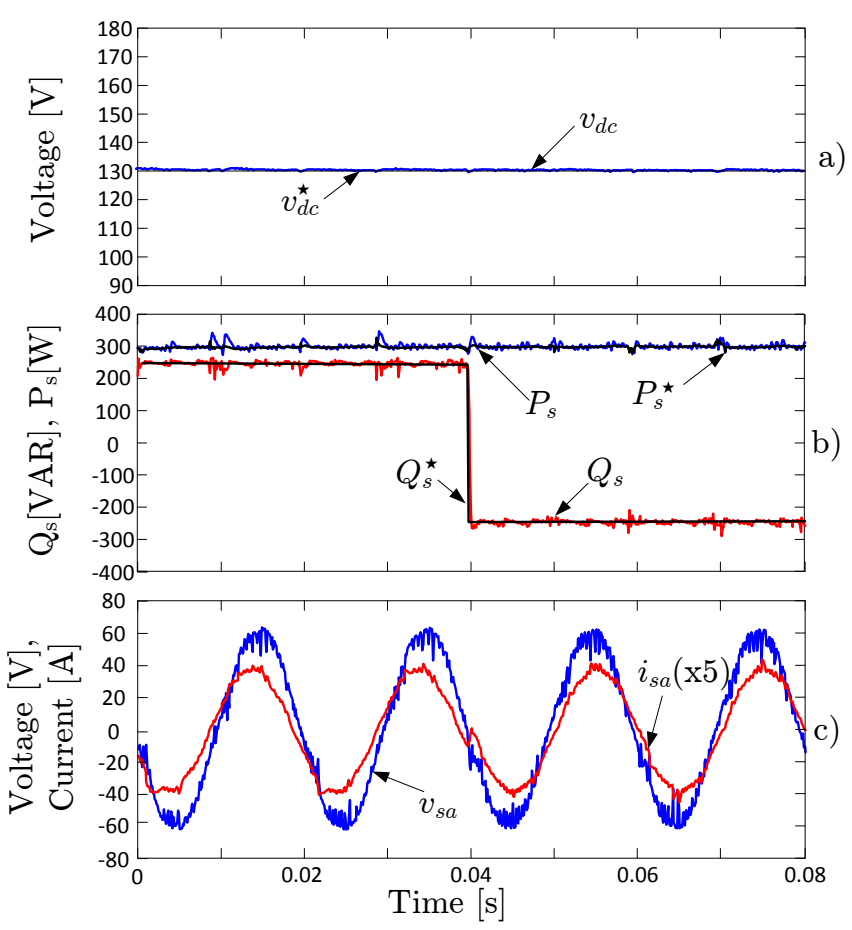

Figure 8. Performance of the MPC algorithm presented - Experiment: step in the reactive power reference $Q_{s}^{\star}$.

\section{Load Changes}

In practical applications, loads may be time-varying and, thus, $R_{d c}$ needs to be estimated. Since estimators will unavoidably be affected by errors, another important aspect to be analyzed is robustness to unknown load changes. Experimental results are depicted in Fig. 9. Here we chose the same initial condition as in Section $\mathrm{V}-\mathrm{A}$, namely, $Q_{s}=0[\mathrm{VAr}]$, and $v_{d c}=130[V]$, which amounts to an active power of about $P_{s}=300[W]$.

At the instant $t=0.1[\mathrm{~s}]$, a resistance load change of $50 \%$ is produced from $R_{d c}=60[\Omega]$ to $R_{d c}=30[\Omega]$. It can be observed in Fig. 9 that the predictive controller proposed was capable to keep the DC-voltage, $v_{d c}$, near its reference value $v_{d c}^{\star}=130[V]$, despite the load disturbance. It is worth noting that the controller has no knowledge of the load disturbance. The system model used to predict the future behaviour simply assumes $R_{d c}=60[\Omega]$ at all times. Consequently, a slight increment in the DC-voltage ripple occurs.

\section{Supply Voltage Variations}

The robustness of the proposed control system to supply voltage variations is also analyzed. Results in Fig. 10 show the response of the system when the supply voltage is reduced in $40 \%$. The reference values are $Q_{s}=0[\mathrm{VAr}]$ and $v_{d c}=130[\mathrm{~V}]$. Here, a soft decrement of the voltage source from $v_{s}=55[\mathrm{~V}]$ to $v_{s}=30$ is introduced. The active power presents an increment from $P_{s}=300[W]$ to $P_{s}=330[W]$ to compensate the variation and keep the DC-voltage fixed at it reference. The input current is increased accordingly from $I_{s}=3,5[A]$ rms to $I_{s}=7[A] \mathrm{rms}$. 


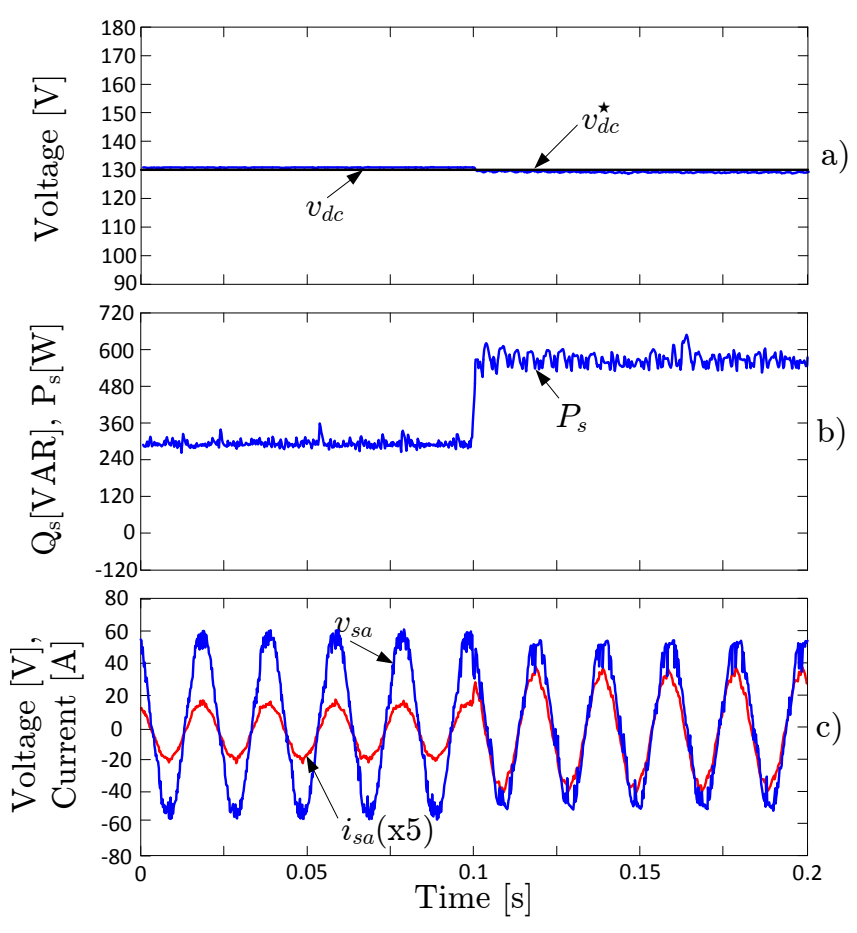

Figure 9. Performance of the MPC algorithm presented - Experiment: step in the load resistance $R_{d c}$.

\section{CONCLusions}

We have presented a model predictive control formulation for AFE rectifiers. The proposed control algorithm operates in discrete-time and does not require any additional modulators to drive the switches. The switching horizon is chosen equal to one. Thus, the search set for on-line optimizations has only eight elements, making the switching signals easy to calculate in practice.

The key novelty of our approach lies in the way dynamic references are handled. To be more specific, careful examination of electrical properties of the rectifier topology has allowed us to elucidate the issue of finding compatible references for active source power and DC-voltage.

Our method is capable of providing suitable references for the source active power and the rectified voltage, without use of additional control loops. The control architecture proposed also incorporates possible saturations of source currents directly in its formulation. This allows one to ensure safe operation of the device. Here the introduction of a reference prediction horizon, which may differ from the switching horizon, has proven useful, allowing the system designer to trade-off tracking bandwidth for control effort.

Simulations and experimental results on a lab-prototype show that fast and accurate tracking of dynamic DC-voltage and reactive power references can be achieved. Interestingly, due to the way that constraints on maximum power levels of the rectifier are treated, good transient performance can be observed even when saturation limits are reached. It is important to emphasize that, during transients where saturations occur, overshoots are avoided.

Future work may include studying robustness of the ap-

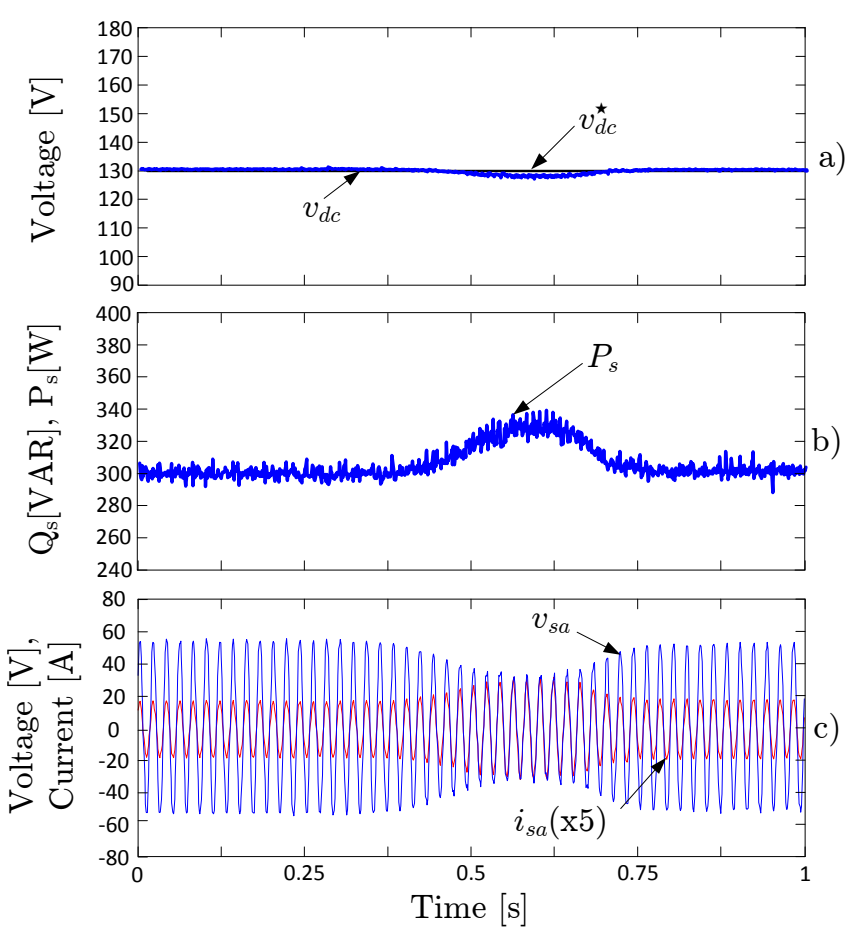

Figure 10. Performance of the MPC algorithm presented - Experiment: supply voltage variation.

proach, adapting the formulation to non-resistive loads, deriving tuning guidelines, and also investigating closed loop stability issues. The latter have been examined for related, but simpler, systems in [33], [34].

\section{REFERENCES}

[1] D. E. Quevedo, R. P. Aguilera, M. A. Pérez, and P. Cortés, "Finite control set MPC of an AFE rectifier with dynamic references," in Proc. IEEE Conf. on Industrial Technology, Valparaíso, Chile, 2010.

[2] J. Dai, D. Xu, B. Wu, and N. R. Zargari, "Unified DC-Link Current Control for Low-Voltage Ride-Through in Current-Source-ConverterBased Wind Energy Conversion Systems," IEEE Trans. Power Electron., vol. 26, no. 1, pp. 288-297, 2011.

[3] J. Dai, D. D. Xu, and B. Wu, "A Novel Control Scheme for CurrentSource-Converter-Based PMSG Wind Energy Conversion Systems," IEEE Trans. Power Electron., vol. 24, no. 4, pp. 963-972, 2009.

[4] Y. W. Li, M. Pande, N. Zargari, and B. Wu, "An Input Power Factor Control Strategy for High-Power Current-Source Induction Motor Drive With Active Front-End," IEEE Trans. Power Electron., vol. 25, no. 2, pp. 352-359, 2010.

[5] J. R. Rodríguez, J. W. Dixon, J. R. Esponoza, J. Pontt, and P. Lezana, "PWM regenerative rectifiers: state of the art," IEEE Trans. Ind. Electron., vol. 52, no. 1, pp. 5-22, Feb. 2005.

[6] S. Kwak and H. A. Toliyat, "Design and rating comparisons of PWM voltage source rectifiers and active power filters for AC drives with unity power factor," IEEE Trans. Power Electron., vol. 20, no. 5, pp. 1133-1142, Sept. 2005.

[7] M. Malinowski, M. P. Kazmierowski, and A. M. Trzynadlowski, "A comparative study of control techniques for PWM rectifiers in AC adjustable speed drives," IEEE Trans. Power Electron., vol. 18, no. 6, pp. 1390-1396, Nov. 2003.

[8] M. P. Kazmierowski, M. Jasinski, and G. Wrona, "DSP-based control of grid-connected power converters operating under grid distortions," IEEE Trans. Ind. Inf., vol. 7, no. 2, pp. 204-211, May 2011.

[9] A. Bouafia, J.-P. Gaubert, and F. Krim, "Predictive direct power control of three-phase pulsewidth modulation (PWM) rectifier using spacevector modulation (SVM)," IEEE Trans. Power Electron., vol. 25, no. 1 , pp. 228-236, Jan. 2010. 
[10] J. Hu, L. Shang, Y. He, and Z. Z. Zhu., "Direct active and reactive power regulation of grid-connected DC/AC converters using sliding mode control approach," IEEE Trans. Power Electron., vol. 26, no. 1, pp. 210-222, Jan. 2011.

[11] C.-C. Hou and P.-T. Cheng, "Experimental verification of the active front-end converters dynamic model and control designs," IEEE Trans. Power Electron., 2011, to appear.

[12] P. Cortés, M. P. Kazmierowski, R. M. Kennel, D. E. Quevedo, and J. Rodríguez, "Predictive control in power electronics and drives," IEEE Trans. Ind. Electron., vol. 55, no. 12, pp. 4312-4324, Dec. 2008.

[13] T. Geyer, G. Papafotiou, R. Frasca, and M. Morari, "Constrained optimal control of the step-down DC-DC converter," IEEE Trans. Power Electron., vol. 23, no. 5, pp. 2454-2464, Sept. 2008.

[14] Y. Xie, R. Ghaemi, and J. S. Freudenberg, "Implicit model predictive control of a full bridge DC-DC converter," IEEE Trans. Power Electron., vol. 24, no. 12, pp. 2704-2713, Dec. 2009.

[15] R. Vargas, U. Ammann, B. Hudoffsky, J. Rodríguez, and P. Wheeler, "Predictive torque control of an induction machine fed by a matrix converter with reactive input power control," IEEE Trans. Power Electron., vol. 25, no. 6, pp. 1426-1438, June 2010.

[16] T. Geyer, "A comparison of control and modulation schemes for medium-voltage drives: Emerging predictive control concepts versus PWM-based schemes," IEEE Trans. Ind. Applicat., vol. 47, 2011, to appear.

[17] A. Linder, R. Kanchan, R. Kennel, and P. Stolze, Model-Based Predictive Control of Electric Drives. Cuvillier Verlag Göttingen, 2010.

[18] S. Kouro, P. Cortés, R. Vargas, U. Ammann, and J. Rodríguez, "Model predictive control-A simple and powerful method to control power converters," IEEE Trans. Ind. Electron., vol. 56, no. 6, pp. 1826-1838, June 2009.

[19] T. Geyer, "Computationally efficient model predictive direct torque control," IEEE Trans. Power Electron., 2011, to appear.

[20] P. Lezana, R. P. Aguilera, and D. E. Quevedo, "Model predictive control of an asymmetric flying capacitor converter," IEEE Trans. Ind. Electron., vol. 56, no. 6, pp. 1839-1846, June 2009.

[21] P. Cortés, J. Rodríguez, D. E. Quevedo, and C. Silva, "Predictive current control strategy with imposed load current spectrum," IEEE Trans. Power Electron., vol. 23, no. 2, pp. 612-618, Mar. 2008.

[22] T. Geyer, G. Papafotiou, and M. Morari, "Model predictive direct torque control-part I: Concept, algorithm and analysis," IEEE Trans. Ind. Electron., vol. 56, no. 6, pp. 1894-1905, June 2009.

[23] P. Cortés, J. Rodríguez, P. Antoniewicz, and M. Kazmierkowski, "Direct power control of an AFE using predictive control," IEEE Trans. Power Electron., vol. 23, no. 5, pp. 2516-2522, Sept. 2008.

[24] K. J. Åström and T. Hägglund, PID Controllers: Theory, Design and Tuning, 2nd ed. Research Triangle Park, N.C.: Instrument Society of America, 1995.

[25] R. P. Burgos, E. P. Wiechmann, and J. Holtz, "Complex state-space modeling and nonlinear control of active front-end converters," IEEE Trans. Ind. Electron., vol. 52, no. 2, pp. 363-377, Feb. 2005.

[26] J. B. Rawlings and D. Q. Mayne, Model Predictive Control: Theory and Design. Madison, WI: Nob Hill Publishing, 2009.

[27] E. F. Camacho and C. Bordons, Model Predictive Control. New York, N.Y.: Springer-Verlag, 1999.

[28] J. M. Maciejowski, Predictive Control with Constraints. Englewood Cliffs, N. J.: Prentice-Hall, 2002.

[29] D. E. Quevedo, C. Müller, and G. C. Goodwin, "Conditions for optimality of naïve quantized finite horizon control," Int. J. Contr., vol. 80, no. 5, pp. 706-720, May 2007.

[30] C. Müller, D. E. Quevedo, and G. C. Goodwin, "How good is quantized model predictive control with horizon one?" IEEE Trans. Automat. Contr., vol. 56, no. 11, pp. 2623-2638, Nov. 2011.

[31] G. C. Goodwin, M. M. Serón, and J. A. De Doná, Constrained Control \& Estimation - An Optimization Perspective. London: Springer Verlag, 2005.

[32] M. Nagahara and D. E. Quevedo, "Sparse representations for packetized predictive networked control," in Proc. IFAC World Congr., Milan, Italy, 2011.

[33] D. E. Quevedo, G. C. Goodwin, and J. A. De Doná, "Finite constraint set receding horizon quadratic control," Int. J. Robust Nonlin. Contr. vol. 14, no. 4, pp. 355-377, Mar. 2004.

[34] R. P. Aguilera and D. E. Quevedo, "On stability and performance of finite control set MPC for power converters," in Proc. Workshop on Predictive Control of Electrical Drives and Power Electronics, Munich, Germany, 2011.

[35] A. Bemporad, "Reference governor for constrained nonlinear systems," IEEE Trans. Automat. Contr., vol. 43, no. 3, pp. 415-419, 1998.
[36] J. A. De Doná, G. C. Goodwin, and M. M. Serón, "Anti-windup and model predictive control: Reflections and connections," European J. Contr., vol. 6, no. 5, pp. 467-477, 2000. 